\title{
ADMX SLIC: Results from a Superconducting LC Circuit Investigating Cold Axions
}

\author{
N. Crisostd* \\ University of Florida, Gainesville, FL 32611, USA and \\ presently at University of Washington \\ P. Sikivie, N. S. Sullivan, and D. B. Tanner \\ University of Florida, Gainesville, FL 32611, USA \\ J. Yang and G. Rybka \\ University of Washington, Seattle, WA 98195, USA
}

(ADMX Collaboration) ${ }^{\dagger}$

(Dated: November 15, 2019)

\begin{abstract}
Axions are a promising cold dark matter candidate. Haloscopes, which use the conversion of axions to photons in the presence of a magnetic field to detect axions, are the basis of microwave cavity searches such as the Axion Dark Matter eXperiment (ADMX). To search for lighter, low frequency axions in the sub $2 \times 10^{-7} \mathrm{eV}(50 \mathrm{MHz})$ range, a tunable lumped-element LC circuit has been proposed. For the first time, through ADMX SLIC (Superconducting LC Circuit Investigating Cold Axions), a resonant LC circuit was used to probe this region of axion mass-coupling space. The detector used a superconducting LC circuit with piezoelectric driven capacitive tuning. The axion mass and corresponding frequency range $1.7498-1.7519 \times 10^{-7} \mathrm{eV}(42.31-42.36 \mathrm{MHz})$, $1.7734-1.7738 \times 10^{-7} \mathrm{eV}(42.88-42.89 \mathrm{MHz})$, and $1.8007-1.8015 \times 10^{-7} \mathrm{eV}(43.54-43.56 \mathrm{MHz})$ was covered at magnetic fields of $4.5 \mathrm{~T}, 5.0 \mathrm{~T}$, and $7.0 \mathrm{~T}$ respectively. Exclusion results from the search data, for coupling below $10^{-12} \mathrm{GeV}^{-1}$ are presented.
\end{abstract}

The constituents of the dark matter of our Universe are yet to be accounted for. Axions are a well-motivated dark matter candidate as they arise independently from the Peccei-Quinn solution to the strong CP Problem [13. If the axion mass is in the $10^{-6}$ to $10^{-5} \mathrm{eV}$ range, therefore very long-lived and very weakly coupled, then the cavity haloscope [4, 5, appears currently the best detection method. The scheme is based on the electromagnetic coupling of axions to two photons:

$$
\mathcal{L}_{a \gamma \gamma}=-g a(x) E \overrightarrow{(x)} \cdot \overrightarrow{B(x)}
$$

where $g$ is a coupling constant, $a$ is the axion field, $\vec{E}$ is the electric field, and $\vec{B}$ is the magnetic field. In the presence of a strong magnetic field, an axion may convert into a real, detectable photon. A tuned resonator can subsequently enhance detection of the axion-sourced photon signal. The axion mass is unknown and the detector must be tuned through a range of possible axion masses. The frequency of the axion sourced photon signal, $\omega$, is set by the condition $\hbar \omega \approx m_{a} c^{2}+\frac{1}{2} m_{a} v^{2}$, where $m_{a}$ is the axion mass and $v$ is axion velocity. The KSVZ [6] and DFSZ [7 models are typically used to set $g_{\gamma}$, where $g=g_{\gamma} \frac{\alpha}{\pi f_{a}}$ and $f_{a}$ is the axion decay constant.

Microwave cavity searches, including RBF 8, UF [9], ADMX [10 14], and HAYSTAC [15] have already

\footnotetext{
* Correspondence to nmc25@uw.edu

$\dagger$ All of the authors also are members of the ADMX collaboration and the work reported reflects results obtained by those authors who worked directly on this project related to but distinct from ADMX.
}

scanned sections of axion parameter space. To complement excluded axion mass parameter space, and probe couplings weaker than past helioscope searches [16, an $\mathrm{LC}$ circuit resonant structure in a lumped element regime can be used instead of a microwave cavity 17-19, and was done in the pilot experiment, ADMX SLIC, presented here. This strategy in resonant structure, essentially a new class of contralto haloscopes, is also being pursued by ABRACADABRA [20, BEAST [21, and the Dark Matter Radio Experiment [22. An optically pumped magnetometer readout system has also been proposed 23.

If the axion exists, Maxwell's equations are modified to become

$$
\begin{gathered}
\nabla \cdot \vec{E}=g \vec{B} \cdot \nabla a \\
\vec{\nabla} \times \vec{B}-\frac{\partial \vec{E}}{\partial t}=g\left(\vec{E} \times \vec{\nabla} a-\vec{B} \frac{\partial a}{\partial t}\right) .
\end{gathered}
$$

In the presence of a strong static magnetic field $B_{0}$, there is an axion-sourced current

$$
\overrightarrow{j_{a}}=-g \overrightarrow{B_{0}} \frac{\partial a}{\partial t}
$$

Thus, there is a detectable oscillating magnetic field

$$
\vec{\nabla} \times \overrightarrow{B_{a}}=\overrightarrow{j_{a}}
$$

in the quasi-static limit, when the length scale over with the external field $\overrightarrow{B_{0}}$ extends is much smaller than $c / \omega$. 
A loop antenna is used to capture the resulting magnetic flux

$$
\Phi_{a}=-V_{m} g \frac{\partial a}{\partial t} B_{0}
$$

where $V_{m}=\frac{1}{4} l r^{2}$ is set by the geometry of the loop antenna with height $l$ and width $r$. Using the definition of inductance it follows that

$$
I_{a}=-\frac{\Phi_{a}}{L_{1}}
$$

in the limit of infinite capacitance. Including the resonant enhancement results in

$$
I_{a}=\frac{Q}{L_{1}} V_{m} g \frac{\partial a}{\partial t} \overrightarrow{B_{0}}
$$

where $Q$ is the quality factor of the circuit. In the case of the experiment described here, $I_{a}$ is inductively coupled to the input of a first-stage amplifier. Flux in the coupling probe from mutual inductance, $M$, is given by

$$
\Phi_{p}=M I_{a}=\kappa \sqrt{L_{1} L_{2}} I_{a}
$$

where $L_{1}$ is the total inductance of the loop antenna and environment parasitics; $L_{2}$ is the coupling probe inductance, giving a signal power of

$$
P_{\text {input }}=\omega \kappa^{2} L_{1} I_{a}^{2}
$$

where $\kappa$ is a coupling constant between $L_{1}$ and $L_{2}$. The pilot experiment ADMX SLIC probes a lower-frequency, lighter-axion mass parameter space that is otherwise difficult to reach with existing microwave-cavity axion haloscope searches. Prototype optimization studies led to a NbTi loop antenna capacitively tuned by a piezoelectricactuated-dielectric deployed at $4.2 \mathrm{~K}$ in magnetic fields of $4.5-7 \mathrm{~T}$.

The loop antenna inductor of the LC circuit used in our axion search was a single rectangular loop, $7.62 \mathrm{~cm}$ $\times 31.25 \mathrm{~cm}$, with copper-matrix-free $0.25 \mathrm{~mm}$ diameter $\mathrm{NbTi}$ wire strung around a polytetrafluoroethylene (PTFE) frame. A parallel capacitor was made with 5.08 $\mathrm{cm} \times 5.72 \mathrm{~cm}$ NbTi plates, supported by PTFE blocks, and positioned with a $0.464 \mathrm{~cm}$ gap between plates. PTFE screws fastened the form and secured the capacitor plates in place. Angle bracket shapes were used to reduce weight and increase stability. A groove in the PTFE was used to secure most of the NbTi wire; PTFE tape secured the horizontal runs. The NbTi capacitor plates were spot welded to the ends of the NbTi loop. Tuning was achieved by moving an alumina sheet between the parallel plate capacitor with a rotary piezoelectric motor (ANR240). The calculated capacitance was $7.86 \mathrm{pF}$ and $5.53 \mathrm{pF}$ with and without the alumina fully inserted respectively. Varactor tuning was previously explored here [24, 25], but was rejected due to observed high insertion loss. Based on the loop antenna dimensions, the Terman formula [26] estimates an inductance of $0.968 \mu \mathrm{H}$.
Given the measured resonant frequency of $42 \mathrm{MHz}$ with the capacitor tuned to its highest value, an effective total inductance, including environment parasitics, of 1.8 $\mu \mathrm{H}$ is found. The loop antenna is directly mounted to a ${ }^{3} \mathrm{He}$ refrigerator. This placement allows for option of increased cooling in future runs and gives a representation of the resonator performance in situ of progressive cryogenic infrastructure.

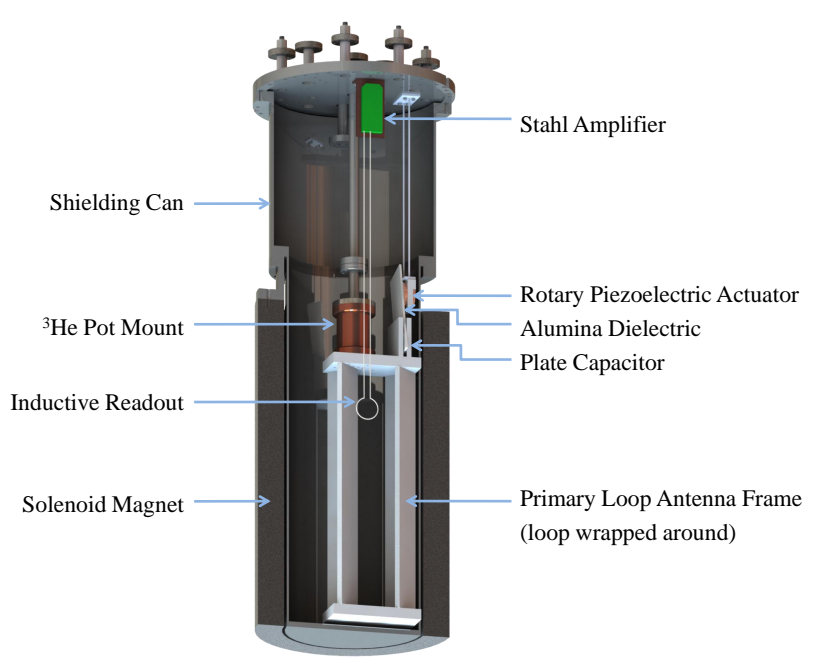

FIG. 1: A sketch of the experiment layout. Two additional weakly coupled probes, for $\mathrm{S}_{21}$ measurements and optional amplifier bypass, are not shown.

The magnet used is a Cryomagnetics superconducting (NbTi) solenoid with a $17.1 \mathrm{~cm}$ bore and a length of 40 $\mathrm{cm}$. The central field is rated to $8.6 \mathrm{~T}$ at a current of $88 \mathrm{~A}$, providing an average field of $7.5 \mathrm{~T}$ in a volume of about 8 liters. In the course of data operations the vacuum can was not completely sealed to allow for cooling through immersion from ${ }^{4} \mathrm{He}$. The magnet is supported from a custom made stainless steel vacuum can. The entire insert is then housed in a super-insulating cryostat made by Precision Cryogenic System, Inc. An an outline of the arrangement can be seen in Figure 1 . The stainless steel vacuum can is lined with $0.003 " \mathrm{Nb}_{45} \mathrm{Ti}_{55}$ sheet for shielding of the loop antenna.

A five turn NbTi, PTFE insulated coil was installed as an inductive coupling of the primary loop antenna to first-stage amplifier input. NbTi leads of the inductive pickup were also connected to the amplifier input by crimping in $\mathrm{CuNi}$ capillary and soldering with $\mathrm{PbSn}$. The highest $Q$ obtained, $Q>10$, 000, was in this configuration.

A low noise cryogenic GaAs FET amplifier 27] is used as the first-stage amplifier. The output of the first-stage cryogenic amplifier above the vacuum can lid is connected via tinned copper braid PTFE insulated coaxial cable to room temperature [28. After post amplification, the signal is fed to a Mini-Circuits ZX05-1-S double balanced mixer 29 and heterodyned to $10 \mathrm{kHz}$. An HP $856 \mathrm{~A}$ is used as the local oscillator in mixing, controlled by GPIB 
and LabView drivers. The resulting IF is read by an FFT SR760 Spectrum Analyzer and written to disk. In between tuning and data taking a Field Fox Microwave Analyzer N9916A is used to measure S-parameters. A diagram of the receiver chain is shown in Figure2, From

Receiver Chain

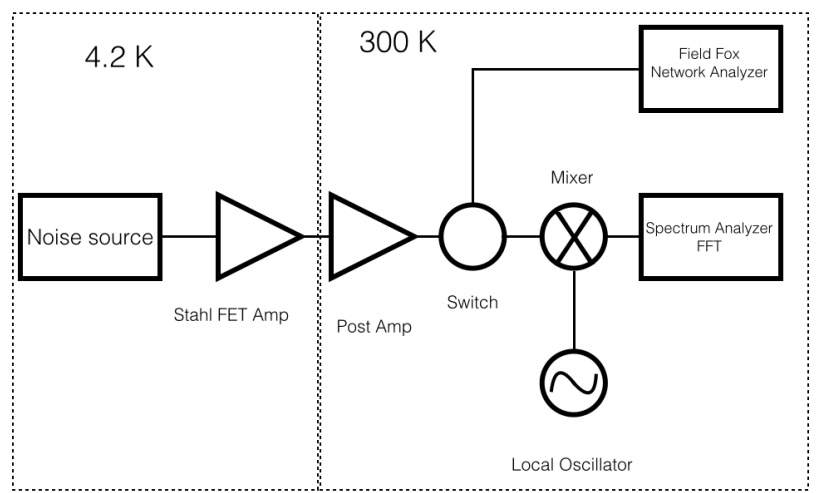

FIG. 2: Receiver chain diagram

the Friis equation [30, the noise temperature of the experiment is calculated to be $19.7 \mathrm{~K}[25]$. The background spectrum analyzer levels corresponded to Johnson voltage noise fluctuations at $23 \mathrm{~K}$. The greater of the two noise temperature figures is used in the reported analysis.

A reduction of $Q$ and frequency shift was found to track the magnet field ramp, as shown in Figure 3. While ramping to $4.5 \mathrm{~T}$, the $Q$ was reduced to $\sim 4,500$. At the highest magnetic fields in data operations, $Q$ 's were typically 2,200-3,200. Similar in field behavior has been reported in other superconducting resonators [31, 32.

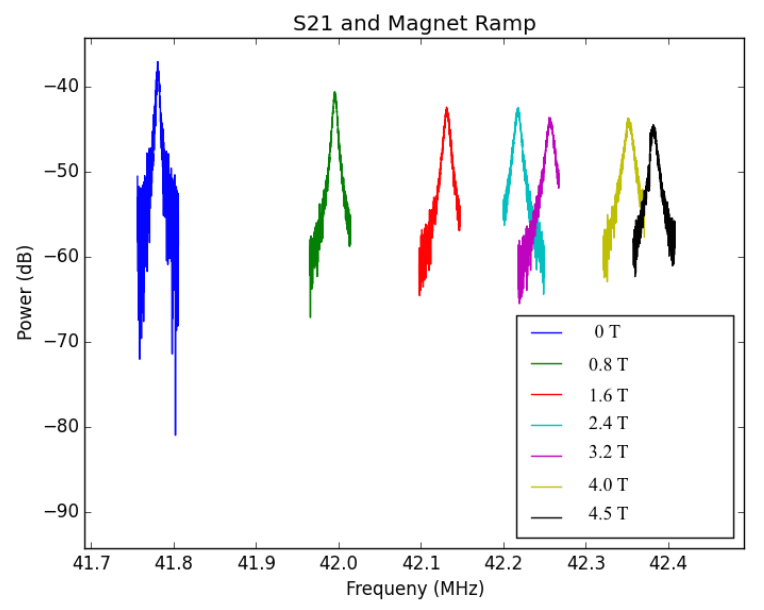

FIG. 3: $\mathrm{S}_{21}$ measurements of the loop antenna taken over the course of a magnet ramp. A frequency shift and $Q$ reduction tracks the increased magnetic field.
Data operations consisted of tuning the LC circuit through its full range. At each frequency step, signal is single heterodyne mixed down to $10 \mathrm{kHz}$ and a FFT spectrum is then taken and digitized with a Stanford Research Systems 760 spectrum analyzer (SRS760). An ANR240 rotary piezoelectric motor actuates a mechanical capacitor for the frequency scanning. Between each tuning step quality factor, resonant frequency, and temperature are measured and recorded.

The FFT spectra were taken with $12.5 \mathrm{kHz}$ spans and $31.25 \mathrm{~Hz}$ wide bins. The SRS760 used takes 400 points per span and has a real time bandwidth of $100 \mathrm{kHz}$. Typically 10,000 averages of $32 \mathrm{~ms}$ scans were taken at each frequency and $1 \mathrm{kHz}$ tuning steps were taken between bouts of spectra collection. A tuning range of $42.3-50.0$ $\mathrm{MHz}$ was expected but in operations was considerably smaller, probably from difficulties with the piezoelectric actuator when cold. Intermittently, synthetic signals were injected, on a weakly coupled probe, and observed to verify data taking operations. Run 1 collected data at $4.5 \mathrm{~T}$ from $6 / 16 / 2018-6 / 19 / 2018$. Run 2 collected data at $5 \mathrm{~T}$ and $7 \mathrm{~T}$ from $7 / 19 / 2018-7 / 27 / 2018$.

The signal-to-noise ratio and expected axion sourced power are used to place limits on the coupling of the axion to two photons from the measured power spectra. The signal-to-noise is calculated from the Dicke radiometer equation 33 using power on the input coil of the firststage amplifier,

$$
S N R=\frac{P_{\text {input }}}{k T} \sqrt{\frac{\Delta t}{\Delta b}}
$$

where $\mathrm{k}$ is Boltzman's constant, $\Delta t$ is integration time, and $\Delta b$ is bandwidth. For each raw spectrum $1 \mathrm{kHz}$ was trimmed off the ends before being co-added to form a grand spectrum. Background subtraction was done through a Savitsky-Golay filter. A significant power excess above average noise power could be indicative of an axion conversion signal [9] and a candidate. A power excess above Gaussian background noise can be calculated from

$$
\# \sigma=\frac{P-\bar{P}}{\bar{P}} \sqrt{N} .
$$

Numerous peaks of power excess were observed in the raw spectra, which are likely radio frequency interference (RFI) from external sources, but were not re-scanned as part of this study. Axion couplings that would produce signals larger than the observed excesses can be excluded, as shown in the blue lines of Fig. 4. Future large scale experiments will be able to implement more shielding and re-scan excesses to distinguish axion signals from RFI; for the purposes of understanding scaling of these experiments, the estimated limits that could have been achieved were RFI candidates eliminated are shown in orange. It is interesting to note that even limited by RFI, unexplored axion-like-particle dark matter parameter space can be excluded. 


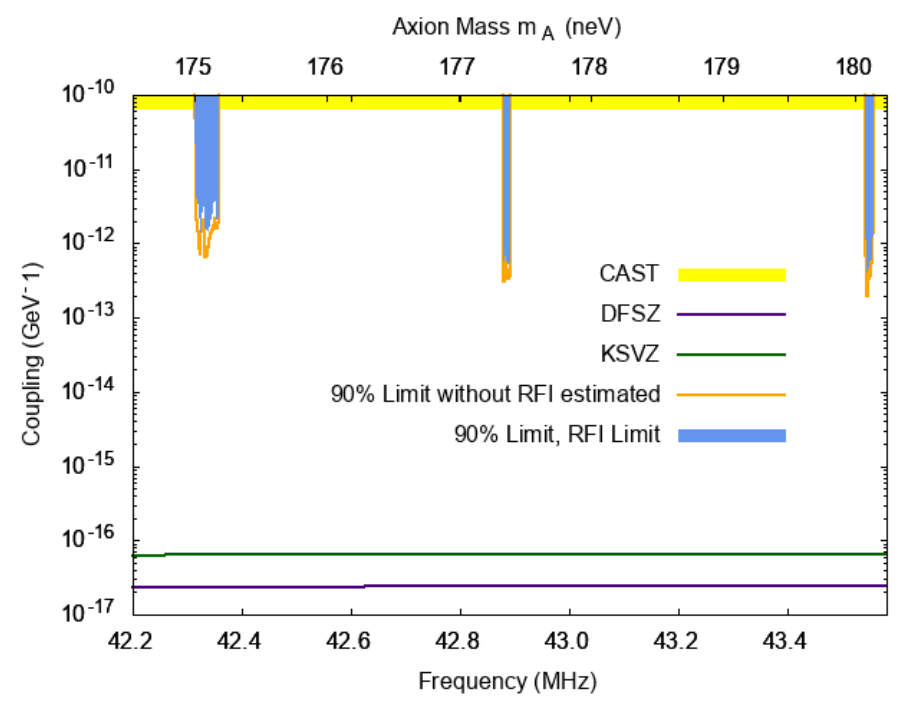

(a) Limit plot from all combined data runs

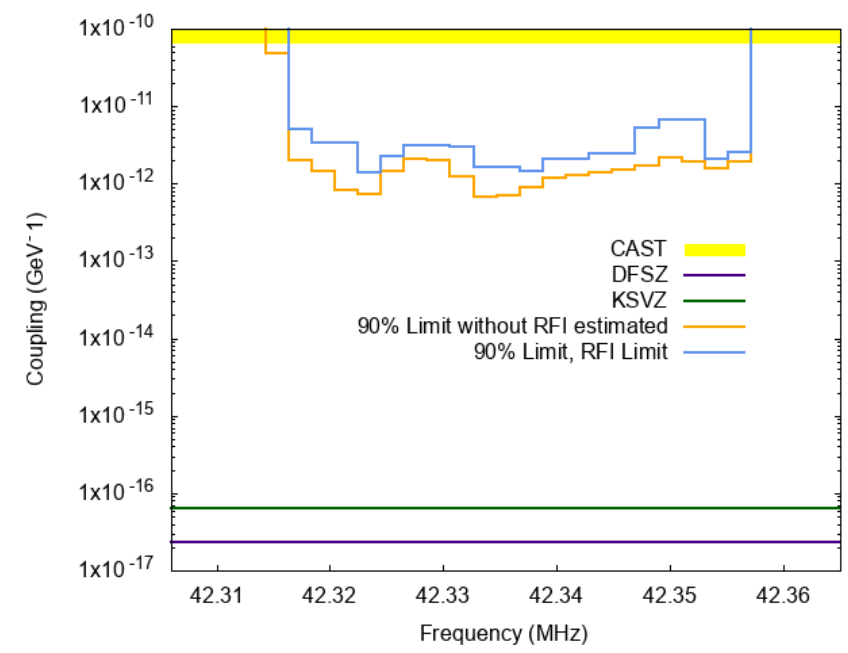

(b) Run 1 with a $4.5 \mathrm{~T}$ magnetic field

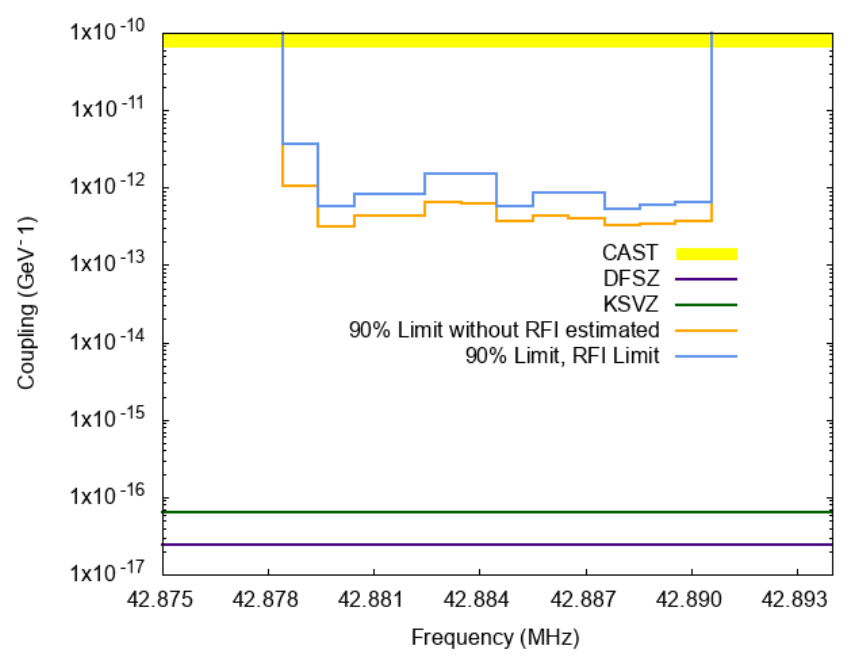

(c) Run 2.a with a 5.0 T magnetic field

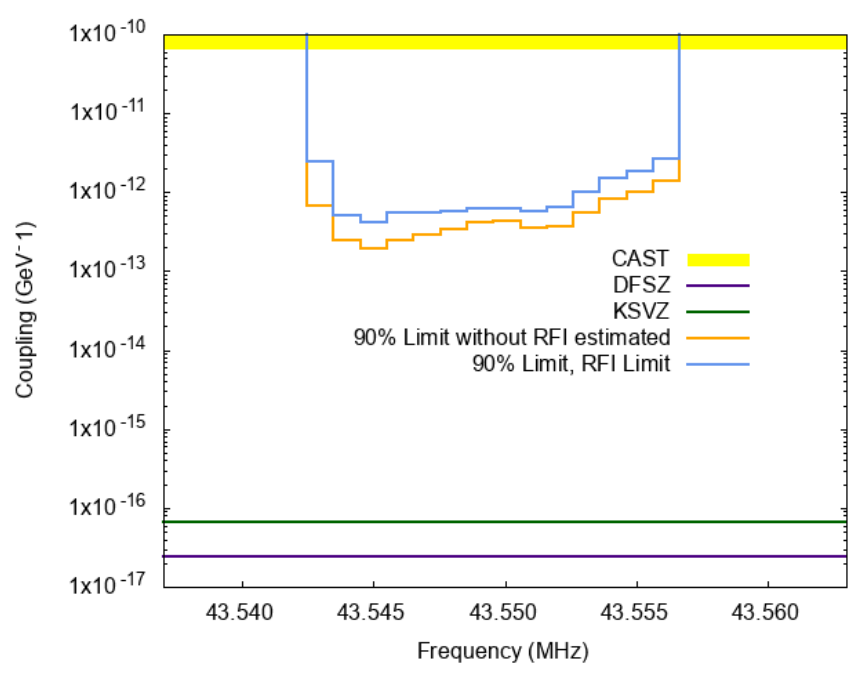

(d) Run 2.b with a $7.0 \mathrm{~T}$ magnetic field

FIG. 4: Individual and combined limits collected by ADMX SLIC 
Figure 4 shows the axion-photon coupling exclusions achieved in the experiment. Panels 4b, 4c, and 4d show the individual runs while panel 4 a shows the regions combined. The previous best limits set by CAST are also shown. The limits presented rule out axions with couplings greater than the limit-line.

An LC circuit based axion search presents a promising approach to scan unexplored regions of low-frequency axion parameter space. Extensive prototype testing culminated in a piezoelectric-actuated dielectric-tuned $\mathrm{NbTi}$ superconducting LC circuit. The operation of this new form of axion detector tested design considerations for future implementations including the measurements of $\mathrm{AC}$ electrical losses in superconducting circuits in a large magnetic field. Our pilot experiment operated in $4.2 \mathrm{~K}$ and magnetic fields of 4.5-7 T. The axion mass range of $1.7498-1.7519 \times 10^{-7} \mathrm{eV}(42.31-42.36 \mathrm{MHz})$,
$1.7734-1.7738 \times 10^{-7} \mathrm{eV}(42.88-42.89 \mathrm{MHz})$, and $1.8007-1.8015 \times 10^{-7} \mathrm{eV}(43.54-43.56 \mathrm{MHz})$ was searched. A large amount of external RF noise was found in the data. Despite an external RFI noise limited analysis a new section of axion mass and coupling was excluded.

Research at the University of Florida has been supported by the US Department of Energy through grants de-sc0010296 and de-sc0009723 TDD. Additional work at the University of Washington is supported by the US Department of Energy through grant de-sc0011665. The authors gratefully acknowledge useful comments by Gianpaolo Carosi, John Clarke, Joe Gleason, Greg Labbe, Bill Malphurs, and Leslie Rosenberg. The authors also gratefully acknowledge the larger ADMX collaboration beyond those responsible for this particular work who provided comments on the draft.
[1] R. D. Peccei and H. R. Quinn, Phys. Rev. Lett. 38, 1440 (1977)

[2] S. Weinberg, Phys. Rev. Lett. 40, 223 (1978).

[3] F. Wilczek, Phys. Rev. Lett. 40, 279 (1978),

[4] P. Sikivie, Phys. Rev. Lett. 51, 1415 (1983)

[5] P. Sikivie, Phys. Rev. D 32, 2988 (1985).

[6] A. Zhitnitsky, Sov.J.Nucl.Phys. 31, 260 (1980).

[7] M. Dine and W. Fischler, Phys. Lett. B 120, 137 (1983)

[8] S. De Panfilis, A. Melissinos, B. Moskowitz, J. Rogers, Y. Semertzidis, et al., Phys. Rev. Lett. 59, 839 (1987).

[9] C. Hagmann, P. Sikivie, N. S. Sullivan, and D. B. Tanner, Phys. Rev. D42, 1297 (1990)

[10] S. J. Asztalos, E. Daw, H. Peng, L. J. Rosenberg, D. B. Yu, C. Hagmann, D. Kinion, W. Stoeffl, K. van Bibber, J. LaVeigne, P. Sikivie, N. S. Sullivan, D. B. Tanner, F. Nezrick, and D. M. Moltz, The Astrophysical Journal Letters 571, L27 (2002)

[11] S. J. Asztalos, G. Carosi, C. Hagmann, D. Kinion, K. van Bibber, M. Hotz, L. J. Rosenberg, G. Rybka, J. Hoskins, J. Hwang, P. Sikivie, D. B. Tanner, R. Bradley, and J. Clarke, Phys. Rev. Lett. 104, 041301 (2010)

[12] J. Sloan, M. Hotz, C. Boutan, R. Bradley, G. Carosi, D. Carter, J. Clarke, N. Crisosto, E. Daw, J. Gleason, J. Hoskins, R. Khatiwada, D. Lyapustin, A. Malagon, S. O'Kelley, R. S. Ottens, L. J. Rosenberg, G. Rybka, I. Stern, N. S. Sullivan, D. B. Tanner, K. van Bibber, A. Wagner, and D. Will, Physics of the Dark Universe 14, 95 (2016)

[13] J. Hoskins, N. Crisosto, J. Gleason, P. Sikivie, I. Stern, N. S. Sullivan, D. B. Tanner, C. Boutan, M. Hotz, R. Khatiwada, D. Lyapustin, A. Malagon, R. Ottens, L. J. Rosenberg, G. Rybka, J. Sloan, A. Wagner, D. Will, G. Carosi, D. Carter, L. D. Duffy, R. Bradley, J. Clarke, S. O'Kelley, K. van Bibber, and E. J. Daw, Phys. Rev. D 94, 082001 (2016).

[14] N. Du, N. Force, R. Khatiwada, E. Lentz, R. Ottens, L. J. Rosenberg, G. Rybka, G. Carosi, N. Woollett, D. Bowring, A. S. Chou, A. Sonnenschein, W. Wester, C. Boutan, N. S. Oblath, R. Bradley, E. J. Daw, A. V. Dixit, J. Clarke, S. R. O'Kelley, N. Crisosto, J. R. Gleason, S. Jois, P. Sikivie, I. Stern, N. S. Sullivan, D. B.
Tanner, and G. C. Hilton (ADMX Collaboration), Phys. Rev. Lett. 120, 151301 (2018).

[15] L. Zhong, S. Al Kenany, K. M. Backes, B. M. Brubaker, S. B. Cahn, G. Carosi, Y. V. Gurevich, W. F. Kindel, S. K. Lamoreaux, K. W. Lehnert, S. M. Lewis, M. Malnou, R. H. Maruyama, D. A. Palken, N. M. Rapidis, J. R. Root, M. Simanovskaia, T. M. Shokair, D. H. Speller, I. Urdinaran, and K. A. van Bibber, Phys. Rev. D 97, 092001 (2018)

[16] V. Anastassopoulos et al. (CAST Collaboration), Nature Physics 13, 584 (2017)

[17] P. Sikivie, N. Sullivan, and D. B. Tanner, Phys. Rev. Lett. 112, 131301 (2014)

[18] P. Sikivie, N. Sullivan, and D. B. Tanner, (unpublished), unpublished work on the LC circuit axion dark matter detector was done in the early 2000's by the authors of ref. [17 and independently by B. Cabrera and S.Thomas. The work of Cabrera and Thomas was presented in a talk.

[19] B. Cabrera and S. Thomas, "Detecting string-scale qcd axion dark matter," (unpublished), 2010 Axions Conference in Gainesville, Florida, January 15-17.

[20] J. L. Ouellet, C. P. Salemi, J. W. Foster, R. Henning, Z. Bogorad, J. M. Conrad, J. A. Formaggio, Y. Kahn, J. Minervini, A. Radovinsky, N. L. Rodd, B. R. Safdi, J. Thaler, D. Winklehner, and L. Winslow, Phys. Rev. Lett. 122, 121802 (2019)

[21] B. T. McAllister, M. Goryachev, J. Bourhill, E. N. Ivanov, and M. E. Tobar, (2018), arXiv:1803.07755 [physics.ins-det]

[22] M. Silva-Feaver, S. Chaudhuri, H. Cho, C. Dawson, P. Graham, K. Irwin, S. Kuenstner, D. Li, J. Mardon, H. Moseley, R. Mule, A. Phipps, S. Rajendran, Z. Steffen, and B. Young, IEEE Transactions on Applied Superconductivity 27, 1 (2017)

[23] P.-H. Chu, L. D. Duffy, Y. J. Kim, and I. M. Savukov, Phys. Rev. D 97, 072011 (2018)

[24] N. Crisosto, P. Sikivie, N. S. Sullivan, and D. B. Tanner, in Microwave Cavities and Detectors for Axion Research, edited by G. Carosi, G. Rybka, and K. van Bibber (Springer International Publishing, Cham, 2018) pp. 127-133. 
[25] N. Crisosto, Searching for Low Mass Axions with an LC Circuit, Ph.D. thesis, University of Florida (2018).

[26] F. Terman, Radio Engineers Handbook (McGraw-Hill, 1950).

[27] Stahl HDC-50.

[28] Pasternak PE-SR405FL.

[29] Minicircuits ZX05-1+.

[30] H. Friis, Proceedings of the IRE 32, 419 (1944).
[31] S. Ulmer, H. Kracke, K. Blaum, S. Kreim, A. Mooser, W. Quint, C. C. Rodegheri, and J. Walz, Review of Scientific Instruments 80, 123302 (2009) https://doi.org/10.1063/1.3271537

[32] M. S. Ebrahimi, N. Stallkamp, W. Quint, M. Wiesel, M. Vogel, A. Martin, and G. Birkl, Review of Scientific Instruments 87, $075110 \quad$ (2016), https://aip.scitation.org/doi/pdf/10.1063/1.4958647.

[33] R. H. Dicke, Rev. Sci. Inst. 17, 268 (1946). 